\title{
Model reduction for active control design using multiple-point Arnoldi methods
}

\author{
G. Lassaux and K. Willcox \\ Aerospace Computational Design Laboratory \\ Massachusetts Institute of Technology \\ Cambridge, MA 02139
}

\section{ABSTRACT}

A multiple-point Arnoldi method is derived for model reduction of computational fluid dynamic systems. By choosing the number of frequency interpolation points and the number of Arnoldi vectors at each frequency point, the user can select the accuracy and range of validity of the resulting reduced-order model while balancing computational expense. The multiplepoint Arnoldi approach is combined with a singular value decomposition approach similar to that used in the proper orthogonal decomposition method. This additional processing of the basis allows a further reduction in the number of states to be obtained, while retaining a significant computational cost advantage over the proper orthogonal decomposition. Results are presented for a supersonic diffuser subject to mass flow bleed at the wall and perturbations in the incoming flow. The resulting reduced-order models capture the required dynamics accurately while providing a significant reduction in the number of states. The reduced-order models are used to generate transfer function data, which are then used to design a simple feedforward controller. The controller is shown to work effectively at maintaining the average diffuser throat Mach number.

\section{INTRODUCTION}

Computational fluid dynamics (CFD) has reached a considerable level of maturity and is now routinely used in many applications for both external and internal flows. Euler and NavierStokes solvers enjoy widespread use for aerodynamic design and analysis, and provide accurate answers for a variety of complex flows. However, despite ever increasing computational power, unsteady problems are computationally very expensive and time-consuming. More efficient methods for time-varying flow can be obtained if the disturbances are small, and the unsteady solution can be considered to be a small perturbation about a steady-state flow [1]. In this case, a set of linearized equations is obtained which can be time-marched to obtain the flow solution at each instant.

Even under the linearization assumption, any CFD-based technique will generate models with a prohibitively high number of states. For this reason, CFD models are not appropriate for many applications where model size and cost are issues. For example, when the aerodynamic solver must be coupled to another disciplinary model, as in aeroelastic analysis or multidisciplinary optimization, CFD models cannot be used. Another application which requires low-order models is control design.
The concept of using active control to enhance the stability properties of an unsteady flow has been addressed for several applications [2],[3]. In order to derive control models that will be effective, it is vital that the relevant unsteady flow dynamics are captured accurately. A model is required that will capture not only the dynamics of the disturbance to be controlled, but also the visibility offered by the sensing and the effect on the flow of the actuation mechanism. A high-fidelity CFD code can offer the degree of flow resolution that is required; however, for control design it is imperative that the flow model have a low number of states.

One approach to creating more efficient flow models is to make a set of physically-based simplifying assumptions about the flow; however, this results in reduced fidelity results. Model reduction is one approach to obtaining efficient, low-order models while retaining the high-fidelity flow dynamics of CFD [4]. The basic idea is to project the high-fidelity CFD solutions onto a set of basis functions which span the flow solution space efficiently. Models are obtained which retain the high-fidelity aerodynamics of the CFD analysis, but which have only a few states. The proper orthogonal decomposition (POD) technique has been developed as an effective method of deriving the basis functions [5], [6] and has been widely applied to many different problems, including the derivation of active control models for vortex shedding [7], [8].

Another set of reduction techniques exist, which derive reduced-order models based on matching moments of the system transfer function. In particular, the Arnoldi algorithm can be used to generate a set vectors which forms an orthonormal basis for the Krylov subspace. Arnoldi-based reduced-order models have been developed for RLC circuits [9] and compressor aeroelastic models [10]. One can liken the momentmatching techniques to matching the transfer function and its derivatives at a particular frequency (usually zero). If $q$ basis vectors are included in the reduced-order model, then $q$ moments of the transfer function will be matched. Because the vectors are derived at a single frequency point, the Arnoldi approach is computationally much cheaper than the POD. Multiple frequency point Arnoldi methods have also been developed [11]. The idea here is to select multiple frequency points about which to match moments. Once again, multiple vectors are evaluated at each frequency point, and the number of moments matched at each point is equal to the number of vectors included in the basis. The computational expense of deriving 
the model increases with the number of frequency points used; however, using several interpolation points typically reduces the size of the resulting reduced-order model. The multiplepoint Arnoldi method provides a way to trade computational expense of model derivation with the size of the reduced-order model.

In this work, reduced-order models will be generated for the flow through an actively-controlled supersonic diffuser. This diffuser is subject to perturbations in the incoming flow. Active control to stabilize the inlet is effected through a bleed actuation mechanism and sensing of upstream pressure. The model will be developed in the time domain and cast in state-space form, which allows for ease of coupling to the active control model.

In this paper, a description of the physical problem will be given and the underlying CFD model will be described briefly. The basic Arnoldi-based reduction algorithm will then be presented, and an extended algorithm which uses multiple frequency points will be developed. Results will be presented for the supersonic diffuser, and a comparison is made with the POD method. Finally, a design of a simple controller for the diffuser is considered.

\section{Computational Model}

\section{A. Nonlinear Aerodynamic Model}

The unsteady, two-dimensional flow of an inviscid, compressible fluid is governed by the Euler equations. The usual statements of mass, momentum, and energy can be written in integral form as

$$
\begin{array}{r}
\frac{\partial}{\partial t} \iint \rho d V+\oint \rho \vec{Q} \cdot d \vec{A}=0 \\
\frac{\partial}{\partial t} \iint \rho \vec{Q} d V+\oint \rho \vec{Q}(\vec{Q} \cdot d \vec{A})+\oint p d \vec{A}=0 \\
\frac{\partial}{\partial t} \iint \rho E d V+\oint \rho H(\vec{Q} \cdot d \vec{A})+\oint p \vec{u} \cdot d \vec{A}=0
\end{array}
$$

where $\rho, \vec{Q}, H, E$, and $p$ denote density, flow velocity, total enthalpy, energy, and pressure, respectively.

The governing equations are discretized using a finite volume formulation on a structured grid. This formulation does not make use of the usual set of conserved variables $\left(\rho, \rho Q_{x}, \rho Q_{y}, E\right)$. Instead, the local flow velocity components $q$ and $q^{\perp}$ are defined using the computational grid. $q$ is the projection of the flow velocity on the meanline direction of the grid cell, and $q^{\perp}$ is the normal-to-meanline component. To simplify the implementation of the integral energy equation, total enthalpy is also used in place of energy. The vector of unknowns at each node is therefore

$$
\mathbf{U}_{i}=\left[\rho_{i}, q_{i}, q_{i}^{\perp}, H_{i}\right]^{T} .
$$

Details of this CFD formulation can be found in Drela [12].

The CFD formulation described above will be applied to unsteady analysis of a supersonic diffuser. Two physically different kinds of boundary conditions exist: inflow/outflow conditions, and conditions applied at a solid wall. At a solid wall, the usual no-slip condition of zero normal flow velocity is easily applied as $q^{\perp}=0$. In addition, we will allow for mass addition or removal (bleed) at various positions along the wall. The bleed condition is also easily specified. We set

$$
q^{\perp}=\frac{\dot{m}}{\rho},
$$

where $\dot{m}$ is the specified mass flux per unit length along the bleed slot. At inflow boundaries, Riemann boundary conditions are used. For the diffuser problem considered here, all inflow boundaries are supersonic, and hence we impose inlet vorticity, entropy and Riemann's invariants. At the exit of the duct, we impose outlet pressure. Variations in inlet and exit conditions will be represented by the disturbance parameter vector $\mathbf{d}$.

The discrete nonlinear system of equations can be written

$$
\mathbf{F}(\dot{\mathbf{U}}, \mathbf{U}, \dot{\mathbf{m}}, \mathbf{d})=0,
$$

where $\mathbf{U}$ contains the state vector at every node as defined in equation (4), $\dot{\mathbf{m}}$ contains the bleed mass fluxes at each bleed location, and $\mathbf{d}$ contains the inlet and exit disturbances as described above.

All results presented in this paper are based on a fixed geometry. The actuation mechanism selected is mass flow bleeding; however, wall displacement could have been another possibility. Such a choice would involve modifications of the grid geometry, which in turn would become another perturbation input for system (6).

\section{B. Linearized Aerodynamic Model}

Steady-state solutions can be evaluated by solving the nonlinear set of equations (6) with $\dot{\mathbf{U}}=0$; however, integrating the full nonlinear equation in time for unsteady flows is computationally expensive. If we limit ourselves to the consideration of small amplitude unsteady motions, the problem can be considerably simplified by linearizing the equations [1]. The unsteady flow and bleed input are assumed to be small perturbations about a steady state, $(\overline{\mathbf{U}}, \overline{\mathbf{m}})$, as follows:

$$
\begin{aligned}
\mathbf{U}(t) & =\overline{\mathbf{U}}+\mathbf{U}^{\prime}(t) \\
\dot{\mathbf{m}}(t) & =\overline{\mathbf{m}}+\dot{\mathbf{m}}^{\prime}(t),
\end{aligned}
$$

and the unsteady inflow disturbances contained in $\mathbf{d}$ are assumed to be small. Performing a Taylor expansion about steady-state conditions and neglecting quadratic and higher order terms in the perturbations $\dot{U}^{\prime}$ and $\mathbf{U}^{\prime}$, the linearized form of equation (6) is

$$
\frac{\partial \mathbf{F}}{\partial \dot{\mathbf{U}}} \frac{d \mathbf{U}^{\prime}}{d t}+\frac{\partial \mathbf{F}}{\partial \mathbf{U}} \mathbf{U}^{\prime}+\frac{\partial \mathbf{F}}{\partial \dot{\mathbf{m}}} \dot{\mathbf{m}}^{\prime}+\frac{\partial \mathbf{F}}{\partial \mathbf{d}} \mathbf{d}=0,
$$

where all derivatives are evaluated at steady-state conditions. The above equation can be rewritten

$$
E \frac{d \mathbf{U}^{\prime}}{d t}=A \mathbf{U}^{\prime}+B_{m} \dot{\mathbf{m}}^{\prime}+B_{d} \mathbf{d}
$$

where the definitions of the matrices $E, A, B_{m}$ and $B_{d}$ follow directly from (8). Both $E$ and $A$ are $n \times n$ real matrices. It is important to note that boundary conditions do not involve 
temporal differentiation (although the prescribed value may be time-varying). Therefore, $n_{2}$ rows of $E$ associated with the $n_{2}$ boundary condition equations are populated with zeroes exclusively: $E$ is singular.

To further simplify notation, we define the global input vector $\mathbf{u}=\left[\dot{\mathbf{m}}^{\prime}, \mathbf{d}\right]^{T}$ and write the linearized CFD system as

$$
\begin{aligned}
E \frac{d \mathbf{U}^{\prime}}{d t} & =A \mathbf{U}^{\prime}+B \mathbf{u} \\
\mathbf{y} & =C \mathbf{U}^{\prime}+D \mathbf{u}
\end{aligned}
$$

where $\mathbf{y}$ is a vector containing the output quantities of interest and $C$ and $D$ are matrices containing mean flow contributions to the output evaluation. For the analyses presented here, the vector $\mathbf{y}$ contains pressure sensing upstream of the shock and the average Mach number over the height of the inlet at a given location.

\section{ARnOldi-BASEd MOdEl ORder REDUCtion}

The idea behind developing a reduced-order aerodynamic model is to project the large space used by a high-fidelity CFD model, such as that described in the previous section, onto a lower dimensional space which is characterized by a set of basis vectors. If these vectors are chosen so as to accurately span the solution space, the model behavior can be captured with just a few states. In this way, a low-order, high-fidelity aerodynamic model can be obtained. There are several options available for selecting the basis vectors; we will focus on Arnoldibased methods.

The theory of Arnoldi-based model reduction will first be described for the generic system $(10,11)$ and then extended for the case of singular $E$ matrix. If the set of $q$ orthonormal basis vectors is contained in the columns of the matrix $V$, a $q$ th order approximation to the perturbation solution can be made by assuming

$$
\mathbf{U}^{\prime}(t)=V \hat{\mathbf{U}}(t),
$$

where $\hat{\mathbf{U}}(t)$ is the reduced-order aerodynamic state vector. Substituting this representation of $\mathbf{U}^{\prime}$ into the linearized governing equations (10) and premultiplying the system by $V^{T}$, we obtain the reduced-order system

$$
\begin{gathered}
\hat{E} \frac{d \hat{\mathbf{U}}}{d t}=\hat{A} \hat{\mathbf{U}}+\hat{B} \mathbf{u}, \\
\hat{\mathbf{y}}=\hat{C} \hat{\mathbf{U}}+D \mathbf{u},
\end{gathered}
$$

where $\hat{E}=V^{T} E V, \hat{A}=V^{T} A V, \hat{B}=V^{T} B, \hat{C}=C V$ and $\hat{\mathbf{y}}$ is the output of the reduced-order system.

\section{A. Arnoldi Basics}

One approach to ensuring accurate representation of system dynamics is to try to match the transfer function of the reduced and original systems. Several different matching criteria are possible. Here we describe a process based on matching moments of the transfer function. This approach is described in more detail in Silveira et al. [9] and Willcox et al. [10].

Consider the transfer function $H(s)$ of system (10) and (11):

$$
H(s)=C(s E-A)^{-1} B+D,
$$

This expression can be expanded in a Taylor series about $s=0$, resulting in the following expression:

$$
H(s)=-\sum_{k=0}^{\infty} m_{k} s^{k}+D
$$

where

$$
m_{k}=C\left(A^{-1} E\right)^{k} A^{-1} B
$$

is the $k$ th moment of $H(s)$. One can think of the moments of the transfer function as being analogous to its derivatives evaluated at $s=0$.

The $q$ th-order basis of Arnoldi vectors spans the $q$ th-order Krylov subspace, which is defined for the single-input, singleoutput system $\dot{\mathbf{U}}^{\prime}=A \mathbf{U}^{\prime}+\mathbf{b} u$ as

$$
\mathcal{K}_{q}(A, \mathbf{b})=\operatorname{span}\left\{\mathbf{b}, A \mathbf{b}, A^{2} \mathbf{b}, \cdot, A^{q-1} \mathbf{b}\right\} .
$$

It can be shown that this $q$ th order basis matches the first $q$ moments of the transfer function. For a system with multiple inputs, the Krylov subspace is generated by considering each input in turn:

$$
\begin{aligned}
\mathcal{K}_{q}(A, B)= & \operatorname{span}\left\{\mathbf{b}_{1}, A \mathbf{b}_{1}, \cdot, A^{q-1} \mathbf{b}_{1},\right. \\
& \left.\mathbf{b}_{2}, A \mathbf{b}_{2}, \cdot, A^{q-1} \mathbf{b}_{2}, \cdots\right\},
\end{aligned}
$$

where $\mathbf{b}_{1}, \mathbf{b}_{2}, \cdots$ are the columns of $B$. For our system (10, $11)$, which includes the matrix $E$, the following theorem applies [11].

\section{Theorem 1 (Krylov Subspace Model Reduction)}

If colspan $(V) \subset \mathcal{K}_{q}\left(A^{-1} E, A^{-1} B\right)$ then the reduced order transfer function $\hat{H}(s)=\hat{C}\left(s \hat{E}-\hat{A}^{-1}\right) \hat{B}+D$ matches the first $q$ moments of the original transfer function $H(s)$.

Proof: see Grimme [11].

\section{B. Multiple Interpolation Point Arnoldi Method}

The basic Arnoldi method results in a reduced-order model which matches coefficients of a Taylor series expansion of the full-order transfer function about $s=0$. Although this means that low-frequency dynamics can be captured accurately with just a few reduced-order states, the model cannot be expected to be well-behaved as the disturbance frequency increases, or to accurately capture transient response. As the frequency range of interest increases, Arnoldi-based reduced-order models often require a large number of basis vectors to achieve accurate results.

Instead of matching transfer function moments at zero frequency (the so-called time moments), an interesting extension is to shift the interpolation point to higher frequencies, or to consider multiple interpolation frequencies. Writing $s=$ $s_{0}+s^{\prime}$, the Taylor series expansion of the transfer function (15) about some complex point $s_{0}$ yields

$$
H(s)=-\sum_{k=0}^{\infty} m_{k}^{\prime} s^{\prime k}+D
$$

where

$$
m_{k}^{\prime}=C\left[\left(A-s_{0} E\right)^{-1} E\right]^{k}\left(A-s_{0} E\right)^{-1} B
$$


Equations (20) and (21) are equivalent to (16) and (17), which can be seen by considering an analogous dynamical system in which $A \leftrightarrow\left(A-s_{0} E\right)$ and $s \leftrightarrow s^{\prime}$. The following statement is therefore a corollary of Theorem 1 , as follows.

\section{Corollary 1}

If $\operatorname{colspan}(V) \subset \mathcal{K}_{q}\left(\left(A-s_{0} E\right)^{-1} E,\left(A-s_{0} E\right)^{-1} B\right)$ then the reduced order transfer function $\hat{H}(s)=$ $\hat{C}(s \hat{E}-\hat{A})^{-1} \hat{B}+D$ matches the first $q$ moments about $s_{0}$ of the original transfer function $H(s)$.

Although both real and imaginary shifting have been investigated in literature (see Grimme [11]), we will focus on minimizing the frequency-response error between the reduced and full-order transfer functions, and therefore restrict our consideration to imaginary interpolation points of the form $s_{0}=j \omega_{0}$. The use of complex matrices raises an important implementation concern: our reduced-order model should be consistent with the original system and yield real outputs. The avoidance of a complex basis $V$ is therefore desirable. This is done by choosing $s_{0}$ and $s_{0}^{*}$ pairwise: two Krylov subspaces $\mathcal{K}$ and $\mathcal{K}^{*}$ are constructed, generated from sequences of vectors that are complex conjugates of each other. No additional computational effort is involved in this method, since knowledge of the first sequence of vectors immediately implies knowledge of its conjugate. Moreover, the conjugate Krylov subspace $\mathcal{K}^{*}$ is not explicitly generated. The union space $\mathcal{K} \cup \mathcal{K}^{*}$ is the span of the real and imaginary parts of the vectors of $\mathcal{K}$. Only one set of vectors is computed, and $V$ will be the set of the real and imaginary parts, after orthogonalization. This is best described in the algorithm below for the single interpolation point $s_{0}=j \omega_{0}$.

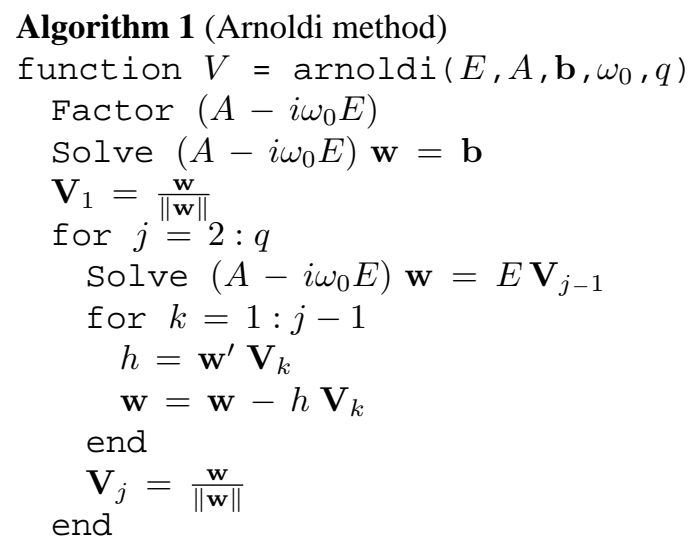

Here, $\mathbf{w}^{\prime} \mathbf{V}_{k}$ denotes the Hermitian product of vectors $\mathbf{w}$ and $\mathbf{V}_{k}$. Each of the $q$ computed vectors is orthogonalized with respect to the previous ones using a Gram-Schmidt orthogonalization process.

The matrix $V$ as computed by Algorithm 1, which contains the $q$ vectors $\mathbf{V}_{j}$ as columns, is an orthonormal complex basis for $\mathcal{K}_{q} . V$ needs to be further processed to span $\mathcal{K}_{q} \cup \mathcal{K}_{q}^{*}$ : the real and imaginary parts of each basis vector will be extracted, and the resulting $2 q$ vectors orthogonalized. There are two possibilities for performing this orthogonalization. GramSchmidt appears to be a natural choice, and can be done at each step of Algorithm 1, in parallel with the Hermitian orthogonalization used for generation of the complex basis. However, an alternate approach is to perform the orthogonalization using singular value decomposition (SVD). Unlike Gram-Schmidt orthogonalization, SVD enables the assessment of the amount of new information added by each basis vector. Using the singular values, the option is available to reduce the size of the resulting basis.

For the sake of simplicity, Algorithm 1 was restricted to a single interpolation point $j \omega_{0}$. This does not yield a better behaved model than the basic Arnoldi model derived about $s=0$, since if a limited number of basis vectors are used, the reduced-order model will give a good match only in the neighborhood of $\omega_{0}$. The range of accuracy of the model can be improved by using multiple interpolation points in order to obtain a good fit over the entire frequency range of interest. In the case of $r$ interpolation points, the basis $V$ spans $\cup_{q=1}^{r} \mathcal{K}_{q}$, which includes $r$ Krylov subspaces by definition. Corollary 1 still holds, and moment matching is thus ensured at every interpolation point $\omega_{0}, \omega_{1}, \ldots, \omega_{r}$. We can therefore evolve Algorithm 1 to consider $r$ frequency points as follows, where $\hat{q}$ is a vector containing the number of Arnoldi vectors to be computed at each interpolation point and $\omega$ is a vector containing the interpolation frequencies.

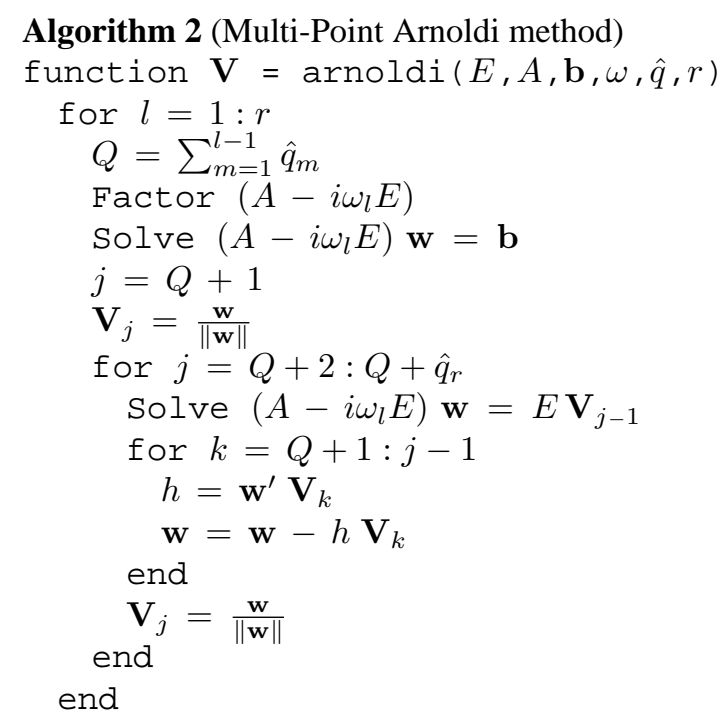

As described previously, real and imaginary parts are then extracted and orthogonalization is done using SVD. At this time, the size of the basis can be further reduced. When the final set of basis vectors, $V$, is obtained, the reduced-order model is constructed by projection as described by equations (13) and (14). This method extends readily to multiple input cases by applying the algorithm to each column of $B$ separately. The final basis is then computed by recombining all the sets obtained from Algorithm 2 with SVD.

\section{Application to Singular Descriptor Matrix Dynamical Sys- tem}

Since the descriptor matrix $E$ is singular, system (10) is algebraic. Although, the previous theorems apply to the singular descriptor matrix system [13], performing reduction via the Arnoldi method directly on system (10) would not only reduce its dynamics but would also affect its boundary conditions. 
However, it is possible to extract an actual state-space system of order $n_{1}=n-n_{2}$, whose dynamics are identical to (10). Boundary conditions will be put aside and treated separately. System (10) can be written

$$
\begin{aligned}
{\left[\begin{array}{cc}
E_{11} & E_{12} \\
0 & 0
\end{array}\right]\left[\begin{array}{c}
\dot{\mathbf{U}}_{1}^{\prime} \\
\dot{\mathbf{U}}_{2}^{\prime}
\end{array}\right]=} & {\left[\begin{array}{ll}
A_{11} & A_{12} \\
A_{21} & A_{22}
\end{array}\right]\left[\begin{array}{c}
\mathbf{U}_{1}^{\prime} \\
\mathbf{U}_{2}^{\prime}
\end{array}\right]+} \\
& {\left[\begin{array}{c}
B_{1} \\
B_{2}
\end{array}\right] \mathbf{u} }
\end{aligned}
$$

where $\mathbf{U}_{2}^{\prime}$ is a vector of length $n_{2}$ containing those flow unknowns which are prescribed via boundary conditions, and the vector $\mathbf{U}_{1}^{\prime}$ comprises the remaining $n_{1}$ unknowns, which are determined by the equations of state.

Since the matrix $A$ is invertible, the change of variables $\mathbf{U}^{\prime}=A^{-1} \mathbf{Z}$ can be applied, yielding

$$
\left[\begin{array}{cc}
\tilde{E}_{11} & \tilde{E}_{12} \\
0 & 0
\end{array}\right]\left[\begin{array}{l}
\dot{\mathbf{Z}}_{1} \\
\dot{\mathbf{Z}}_{2}
\end{array}\right]=\left[\begin{array}{cc}
I_{1} & 0 \\
0 & I_{2}
\end{array}\right]\left[\begin{array}{l}
\mathbf{Z}_{1} \\
\mathbf{Z}_{2}
\end{array}\right]+\left[\begin{array}{c}
B_{1} \\
B_{2}
\end{array}\right] \mathbf{u}
$$

where $\tilde{E}=E A^{-1}$, and $I_{1}$ and $I_{2}$ are the identity matrices of dimensions $n_{1}$ and $n_{2}$ respectively. The prescribed states $\mathbf{Z}_{2}$ can now be condensed out, leading to the actual state-space system

$$
\tilde{E}_{11} \dot{\mathbf{Z}}_{1}=\mathbf{Z}_{1}+B_{1} \mathbf{u}+\tilde{E}_{12} B_{2} \dot{\mathbf{u}}
$$

where we have used $\mathbf{Z}_{2}=-B_{2} \mathbf{u}$. Denoting $\tilde{C}=C A^{-1}=\left[\begin{array}{ll}\tilde{C}_{1} & \tilde{C}_{2}\end{array}\right]$, the expression for the output y becomes

$$
\mathbf{y}=\tilde{C}_{1} \mathbf{Z}_{1}+\left(D-\tilde{C}_{2} B_{2}\right) \mathbf{u}
$$

The multiple frequency point Arnoldi reduction technique described is applied to the condensed state-space system (24, 25). The existence of a 'second' input $\dot{\mathbf{u}}$ does not alter the method described previously, as shown by the transfer function

$$
H(s)=\tilde{C}_{1}\left(s \tilde{E}_{11}-I_{1}\right)^{-1}\left(B_{1}+s \tilde{E}_{12} B_{2}\right)+\tilde{D}
$$

where $\tilde{D}=\left[D-\tilde{C}_{2} B_{2}\right]$. The presence of $\dot{\mathbf{u}}$ simply doubles the number of inputs to consider, since we can define a new set of moments generated by the term $\tilde{E}_{12} B_{2}$. Application of the multiple input, multiple frequency point Arnoldi method described above is unchanged, and the resulting basis spans the following Krylov subspace:

$$
\begin{array}{r}
\mathcal{K}_{q}\left\{\left(I_{1}-j \omega_{0} \tilde{E}_{11}\right)^{-1} \tilde{E}_{11},\right. \\
\left.\left(I_{1}-j \omega_{0} \tilde{E}_{11}\right)^{-1}\left[B_{1}, \tilde{E}_{12} B_{2}\right]\right\} .
\end{array}
$$

Due to the CFD formulation, boundary condition rows are mixed with state equations rows in $\tilde{E}=E A^{-1}$. It would not be practical to explicitly extract $\tilde{E}_{11}$ and $\tilde{E}_{12}$. Instead of computing $V$, an $n_{1} \times q$ matrix, it is far more convenient to compute $\left[\begin{array}{ll}V^{T} & 0\end{array}\right]^{T}$, an $n \times q$ matrix, directly with the original system (10). For details of the actual implementation, see Lassaux [14].

\section{RESULTS}

Reduced-order models have been developed for supersonic started duct flows. A supersonic diffuser has been studied at a steady-state Mach number of 2.2 and a nominal upstream bleed of $1 \%$ of the inlet mass flow. Figure 1 shows the different inputs and outputs of the system. The output of interest is the average Mach number at the throat in response to two unsteady inputs: a planar inlet density perturbation and an upstream bleed perturbation about the nominal bleed. Bleed occurs through small slots located on the lower wall between $46 \%$ and $49 \%$ of the inlet overall length. For control purposes, a sensor is located on the top wall, which measures pressure variations from the steady-state pressure.

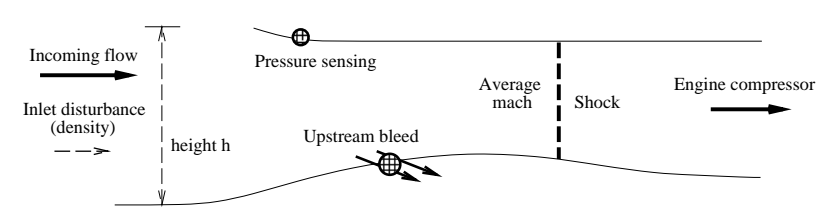

Fig. 1. Definition of the system inputs and outputs: incoming density disturbance, air bleed, pressure sensing, and average Mach number at the throat.

The CFD computational grid has 3078 points, which corresponds to 11,730 unknowns. A time-domain computation of this size is expensive, however we will show that the linearized flow dynamics can be accurately captured with less than 80 modes. Several reduction methods will be shown, all of which are applied over the frequency range 0 to $2 f_{0}$, where $f_{0}=h / a_{0}$ is the reference frequency of the diffuser, defined as its height divided by the freestream speed of sound. According to the atmospheric model considered, disturbances are expected to be in this range.

\section{A. Unshifted Arnoldi Method}

We begin with the basic Arnoldi method, which leads to a basis of vectors that matches moments of the transfer function about $s=0$. Orthogonalization is performed using a GramSchmidt procedure. Figures 2 and 3 show the resulting gains and phases of the transfer functions from bleed input to average throat Mach number, for the full-order and reduced-order systems. In each plot, the dotted line corresponds to the full-order transfer function $(n=11,730)$. The solid lines are transfer functions for reduced-order models of size $q=10,20,30,40$ and 50, plotted from top to bottom. With just ten states in the reduced-order model, a good approximation is obtained locally near $f / f_{0}=0$, however for higher frequencies the error increases rapidly. As more Arnoldi vectors are added to the basis, the quality of the match improves for higher and higher frequencies. With 40 and 50 basis vectors, the transfer function is approximated well over the entire frequency range of interest, although some discrepancy in phase at high frequencies is observed. Although the size of the reduced-order models has increased to obtain this fit, we note that the computational expense to obtain each of the models shown in Figures 2 and 3 is of the same order (i.e. the cost of a single system factorization).

The evolution of accuracy with total number of modes is better shown in Figure 4. In this figure, the absolute value of the error in transfer function gain at each frequency is plotted for 

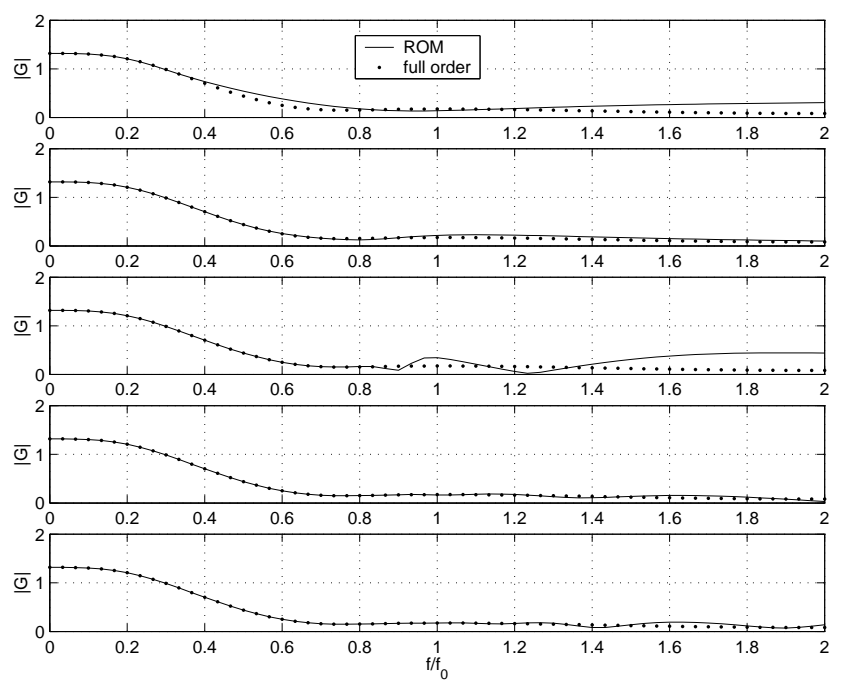

Fig. 2. Magnitudes of full-order transfer function vs. ROM transfer function (bleed to average Mach number at throat). From top: 10, 20, 30, 40 and 50 reduced-order states, all models interpolated at $f / f_{0}=0$.
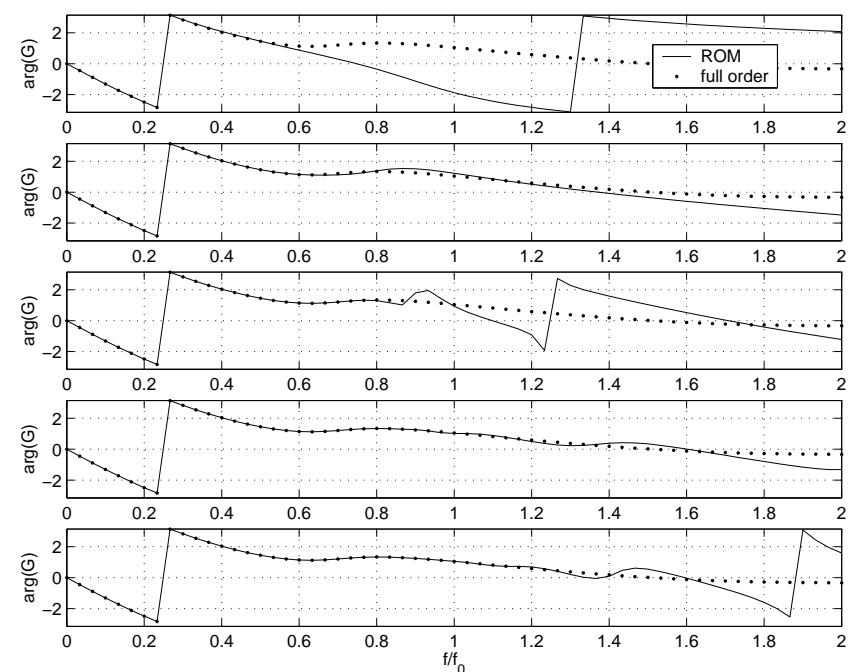

Fig. 3. Phases of full-order transfer function vs. ROM transfer function (bleed to average Mach number at throat). From top: 10, 20, 30, 40 and 50 reducedorder states, all models interpolated at $f / f_{0}=0$.

the reduced-order models shown in Figure 2. It can be seen that with just 10 modes, the model is very accurate in the neighborhood of $f=0$, and this local accuracy does not improve as more modes are added (due to round-off errors). Figure 4 shows clearly that as more modes are included in the model, the accuracy at frequencies away from the interpolation point increases. It is interesting to note that for the $q=30$ case, the improvement in accuracy over the frequency range $\left[0,0.8 f / f_{0}\right]$ is achieved at the expense of a worse prediction at higher frequencies. This can also be clearly seen in Figure 2 and highlights the fact that these basic Arnoldi models can be unreliable far away from their interpolation point. In order for all gain errors over the frequency range to be less than $10^{-2}$, more than 100 basis vectors were required in the reduced-order model.

\section{B. Multiple-Point Arnoldi Method}

While the previous results showed that the basic Arnoldi model required a large number of states to accurately capture the relevant dynamics over the frequency range, significant improvement can be realized by using the multiple-point Arnoldi method. As discussed, using multiple interpolation points causes moments of the transfer function to be matched at several frequencies. At each frequency chosen to be an interpolation point, the fit between the reduced-order and fullorder transfer functions is exact. This can be seen in Figures 5 and 6 where results are shown using five frequency interpolation points: $f / f_{0}=0,0.5,1,1.5$ and 2 . The top plot in both Figures 5 and 6 is a reduced-order model of total size $q=9$. This corresponds to one Arnoldi vector at each frequency point, calculated using Algorithm 2 and orthogonalized using Gram-Schmidt. Note that the Arnoldi vectors at the nonzero frequency points are complex, and thus result in two basis vectors (total number of basis functions $=1+4 * 2=9$ ). As the plot shows, the transfer functions of the reduced and full-order systems match exactly at the selected interpolation points. The local behavior of the moment matching approach can also be seen: for frequencies not close to an interpolation point, the error is large.

The size of the reduced-order model is increased by considering increments of one Arnoldi vector at each interpolation point. This results in reduced-order models of size $q=$ $18,27,36$ and 45 , whose transfer functions are shown in the lower four plots of Figures 5 and 6 from top to bottom respectively. These figures demonstrate the analogy between the moments and the derivatives of the transfer function. As discussed, for the $9^{\text {th }}$-order model (the top plots), the value of transfer function at each interpolation point is matched exactly. In the second plots (two Arnoldi vectors and hence two moments matched per frequency point), it can be seen that the value of the transfer function plus its slope are matched. Figures 5 and 6 also show that as the number of Arnoldi vectors

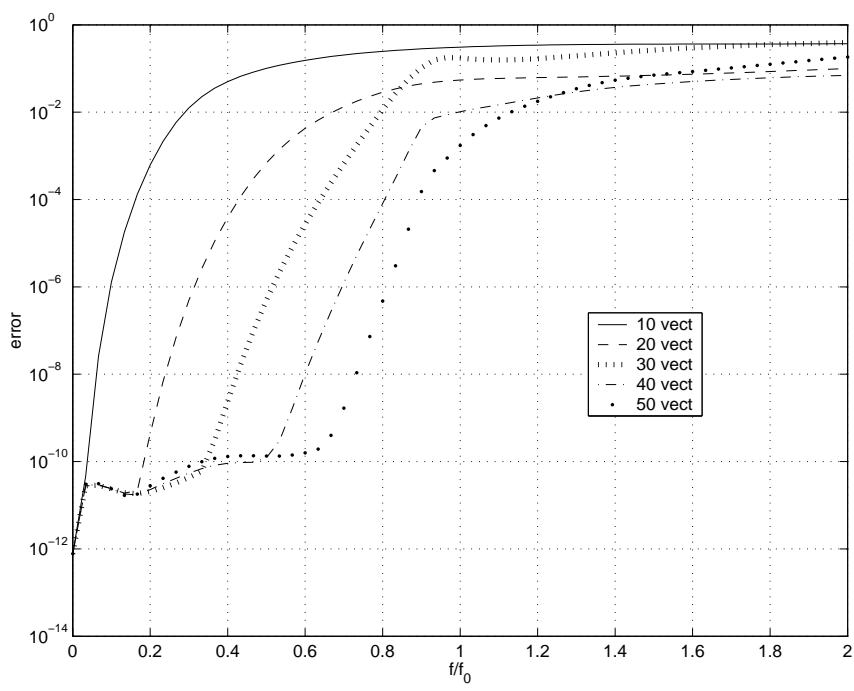

Fig. 4. Absolute value of error in transfer function magnitude for five reducedorder models of size $10,20,30,40,50$. All models interpolated at $f / f_{0}=0$. 
at each interpolation point is increased, the fit away from that frequency improves.

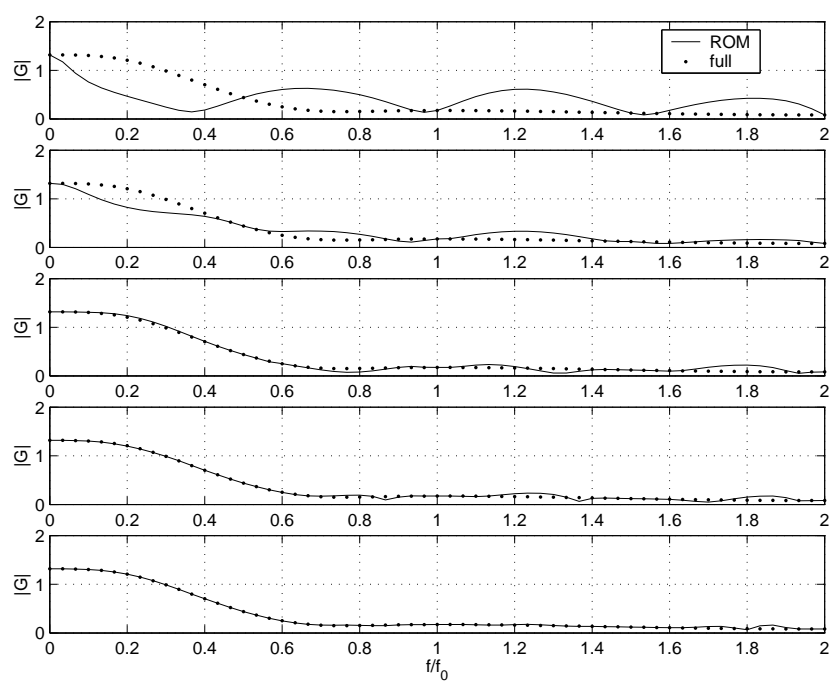

Fig. 5. Magnitudes of full-order transfer function vs. ROM transfer function (bleed to average Mach number at throat). All models derived with five interpolation points at $f / f_{0}=0,0.5,1,1.5,2$. From top: $9,18,27,36$ and 45 reduced-order states.
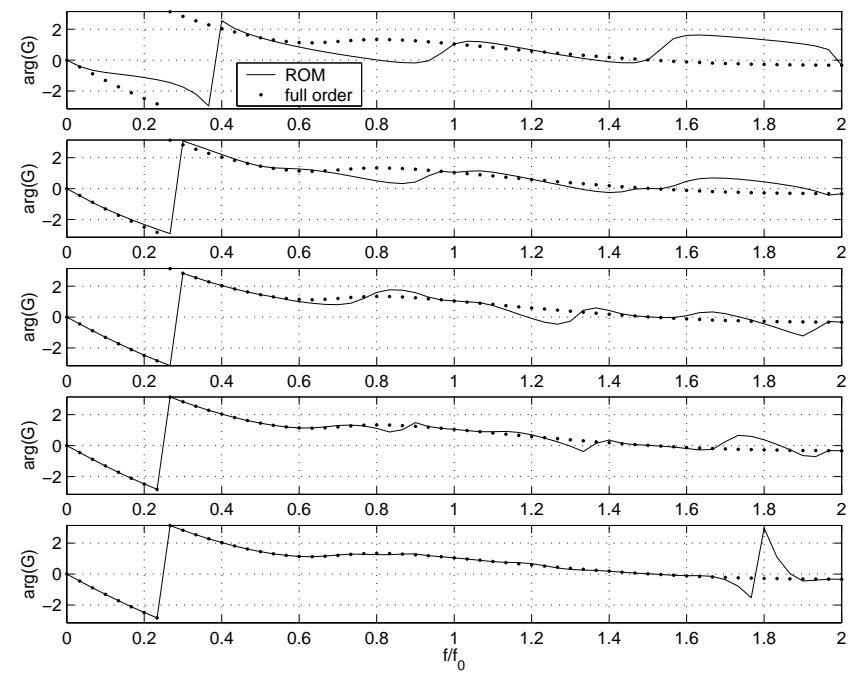

Fig. 6. Phases of full-order transfer function vs. ROM transfer function (bleed to average Mach number at throat). All models derived with five interpolation points at $f / f_{0}=0,0.5,1,1.5,2$. From top: $9,18,27,36$ and 45 reducedorder states.

Figure 7 shows the error in transfer function gain between reduced-order and full-order transfer function versus frequency for five models ranging in size from $q=18$ to $q=63$. For the four lower order models, the maximum error is on the order $10^{-1}$. A significant reduction in error is achieved by including 63 state vectors. In this case, the maximum error over the frequency range of interest is $10^{-2}$. For the single-point Arnoldi method, more than 100 basis vectors are required to achieve this level of accuracy. Figure 7 shows clearly that for a low number of states, accuracy remains localized to the neighborhood of the interpolation points.

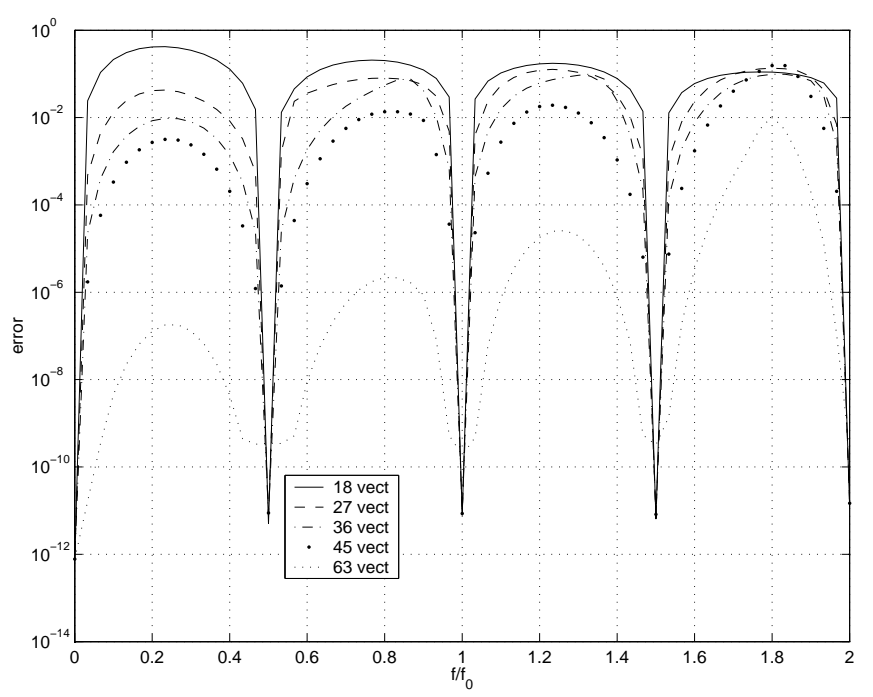

Fig. 7. Absolute value of error in transfer function gain for five reduced-order models of size $18,27,36,45,63$. All models derived with five interpolation points at $f / f_{0}=0,0.5,1,1.5,2$.

Gram-Schmidt was used to orthogonalize all the basis vectors for the multiple-point Arnoldi models described above. A better approach is to use SVD to perform this orthogonalization. Once the Arnoldi basis about each frequency point has been computed (which uses an internal Gram-Schmidt procedure), the resulting sets of vectors can be combined and orthogonalized using SVD. This approach gives us the option to select a subset of the resulting orthogonal vectors, based on the calculated singular values. If not all of the vectors are retained, then the moment matching properties will not be preserved exactly. However, the singular values give a good indication of the importance of a particular mode, and neglecting those modes with small singular values was found to result in efficient reducedorder models without compromising accuracy. Results using SVD will be presented in the following examples.

\section{From Multiple-Point Arnoldi to Proper Orthogonal Decom- position}

Since the efficiency of the reduced-order model has been significantly increased by using the multiple-point Arnoldi method, the question might be asked: why not increase the number of interpolation points and reduce the number of moments matched per interpolation point? As discussed by Willcox et al. [10], as the number of frequency points is increased and the number of moments matched at each point is reduced to one, the method becomes a frequency-domain POD approach, which uses SVD on a set of complex responses obtained at selected frequency sample points to construct a basis [15], [16], [17].

Figure 8 compares the accuracy provided by the multiplepoint Arnoldi method and the POD method. The top plot shows the maximum absolute value of error in the transfer function over the frequency range $\left[0,2 f_{0}\right]$. This error is plotted versus the total number of basis vectors in the reduced-order model for five approaches. The first three models use multiple-point Arnoldi with 5, 11 and 21 interpolation points. The baseline 
models in these three cases contained 99, 126 and 123 basis vectors respectively (corresponding to 11, 6 and 3 Arnoldi vectors per frequency point). SVD was then used to select an orthogonal subset of the baseline vectors and create reducedorder models ranging in size from $q=1$ to $q=75$. The last two models shown in Figure 8 were created using POD with 41 and 61 frequency points. It can be seen that for models of size $q=30$ or less, the maximum error is virtually independent of the number of interpolation points. For higher-order models and lower error levels, the plot shows that, in most cases, choosing more frequency points improves the accuracy of the reduced-order model for a given number of basis functions. (When a very large number of basis vectors is selected, numerical noise becomes an issue, as can be seen for the 61-point POD model.) This improvement in accuracy must be traded with the computational cost of deriving the model, which is directly proportional to the number of interpolation points. The lower plot in Figure 8 shows the first 75 singular values for each of the models. While the POD singular values drop off more rapidly, the plot shows that there is considerable advantage in post-processing the Arnoldi-based models with SVD to obtain a further reduction in size.
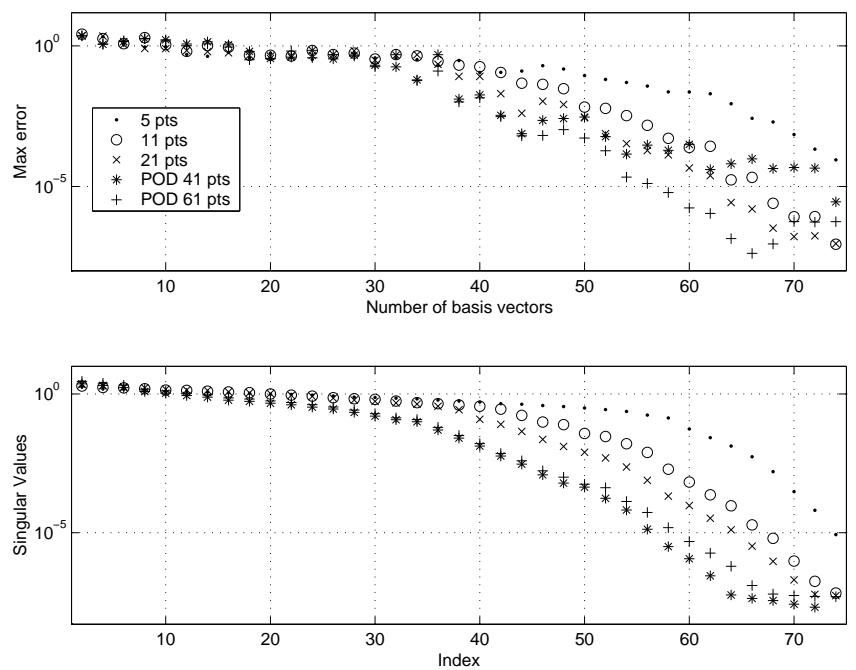

Fig. 8. Comparison of multiple-point Arnoldi and POD models. Results are shown for Arnoldi models with 5, 11 and 21 interpolation points, and POD models with 41 and 61 interpolation points. Top: maximum absolute value of error in transfer function gain over $\left[0,2 f_{0}\right]$ versus number of basis vectors. Bottom: singular values.

The final example demonstrates the accuracy of a multiplepoint Arnoldi model for a time domain computation. For this case, we consider the second unsteady input to the system, an incoming density perturbation. Five interpolation points were used, at frequencies of $f / f_{0}=0,0.5,1,1.5,2$. Ten Arnoldi vectors were calculated at each interpolation point, resulting in a total of 90 vectors. These vectors were then combined and orthogonalized using SVD. The magnitude of the singular values drops off rapidly once the basis reaches a size of approximately $q=70$. Reduced-order models of varying size were constructed using the vectors obtained from the SVD analysis. Figure 9 shows a time-domain simulation of the diffuser in re- sponse to an incoming density perturbation calculated by the linearized CFD code and several reduced-order models. The perturbation considered is constant across the inlet plane, but varies temporally with a Gaussian distribution as follows:

$$
\rho^{\prime}(t)=-0.02 \rho_{0} e^{-\alpha\left(t-2 / f_{0}\right)^{2}} .
$$

The parameter $\alpha$ in the above equation was selected to be $\alpha=$ $6 f_{0}^{2}$, so that the dominant frequency content of the perturbation falls in the range $\left[0,2 f_{0}\right]$. The responses shown in Figure 9 support the conclusion drawn from the distribution of singular values; for models with $q=40$ and $q=50$, there is significant error in the time domain response. Increasing the number of modes to 60 gives a very good result, while the response with 70 modes is virtually indistinguishable from the CFD.

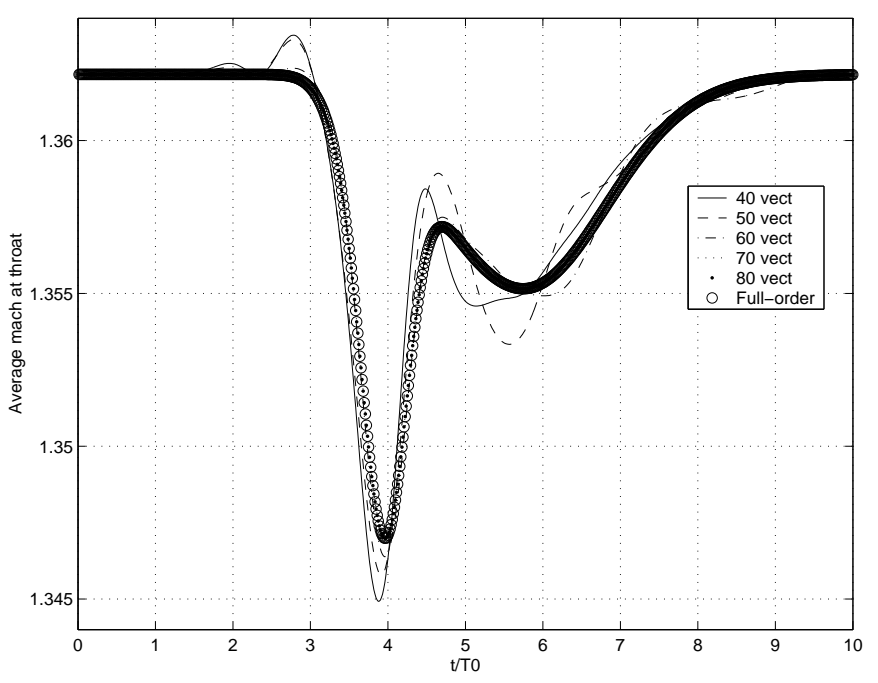

Fig. 9. Linearized response to a density perturbation in the incoming flow. The full-order model (circles) is compared with several reduced-order models ranging in size from 40 to 80 states.

\section{Control Design}

The reduced-order models will now be used to develop a simple controller to monitor the average Mach variation over the throat of the supersonic duct, in response to incoming flow variations. These results will not focus on the specifics of controller design, but rather are intended to demonstrate that a controller designed using reduced-order system dynamics is effective when applied to the full-order model. A feedforward controller is used that evaluates the level of upstream bleed needed to limit the perturbation due to the incoming density disturbance, after assessing its importance via the upstream sensor. The block diagram of the control system is shown in Figure 10. Since the flow upstream of the throat is supersonic, the pressure sensor is not affected by the actuation input.

The relationship between the controlled average Mach number variation $y$ and the incoming density disturbance $d$ can be written as

$$
Y(s)=\left(G+W_{a} W_{c} W_{s}\right) D(s)
$$




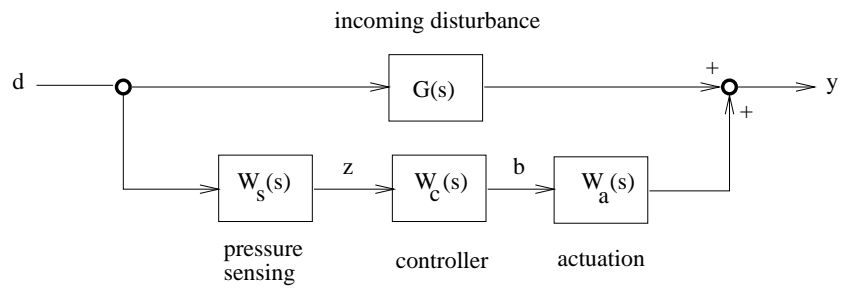

Fig. 10. Block diagram of control system using a feedforward controller.
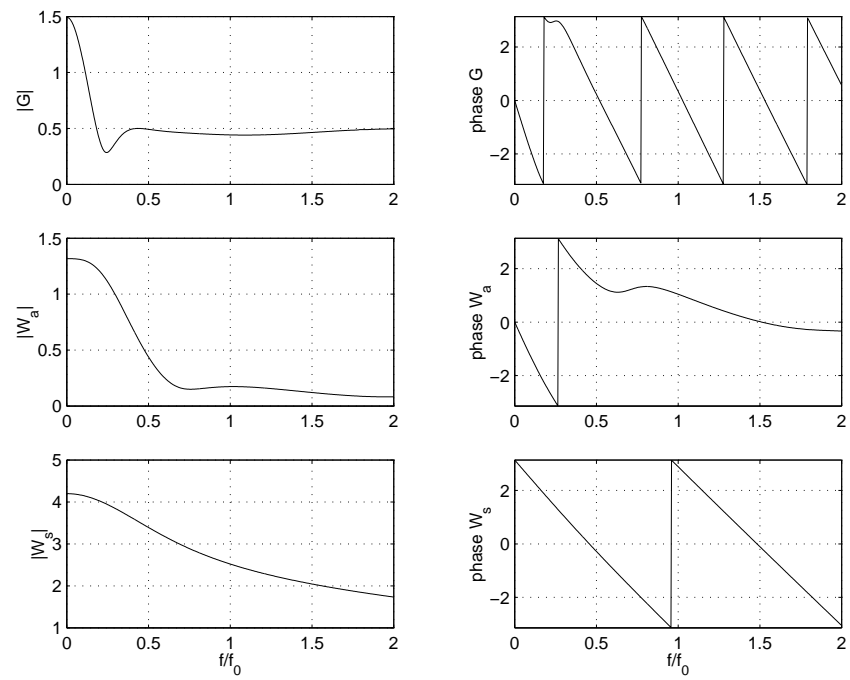

Fig. 11. Gain (left) and phase (right) of the transfer functions of the system. From top to bottom: perturbation to be controlled $G$, actuation $W_{a}$ and sensor $W_{s}$.

where $Y(s)$ and $D(s)$ are the Laplace transforms of the output $y$ and disturbance input $d$ respectively. The transfer functions in (29) are as follows: $G(s)$ represents disturbance input to average throat Mach number, $W_{a}$ represents fractional mass flow input to average throat Mach number, $W_{s}$ represents disturbance input to pressure sensing output, and $W_{c}$ represents the controller (pressure sensing input to mass flow bleed output).

For this study, three reduced-order models were built to simulate the throat Mach number response to an incoming density disturbance, the throat Mach number response due to bleed, and the sensed pressure response due to the inlet density perturbation. For each model, five interpolation points were used, at frequencies of $f / f_{0}=0,0.5,1,1.5,2$. Ten Arnoldi vectors were calculated at each interpolation point, resulting in a total of 90 vectors. Based on results such as those shown earlier, it was determined that including 76 states in the reduced-order models results in sufficient accuracy over the frequency range of interest. The transfer functions $G, W_{a}$ and $W_{s}$ based on the above reduced-order models were computed over the range $\left[0,2 f_{0}\right]$ and are shown in Figure 11.

Once the reduced-order models have been generated, the input/output behavior can be evaluated at many frequencies with low computational cost. Each evaluation requires one system factorization and solve, which for the high-order system is ex-
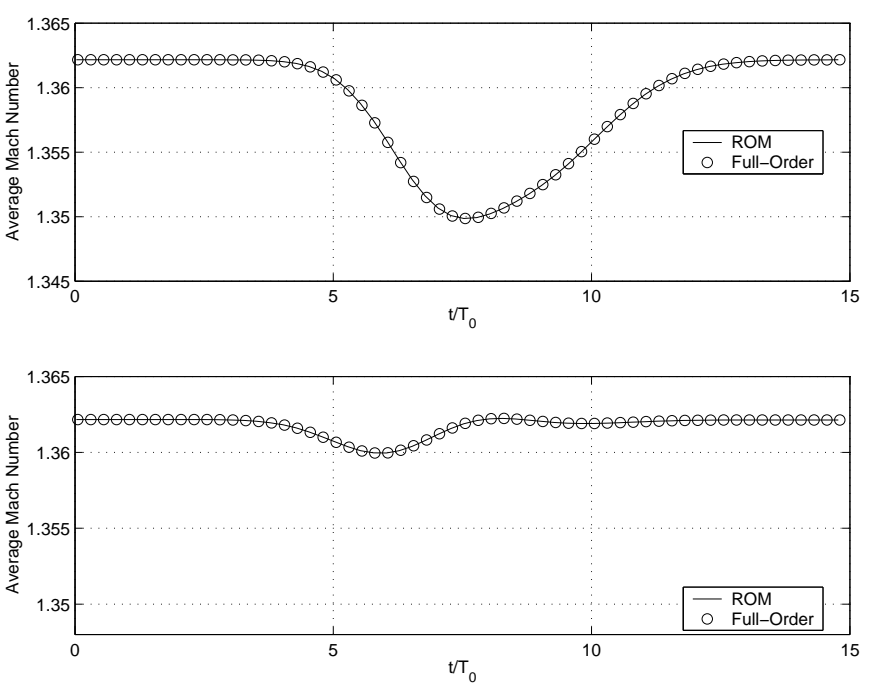

Fig. 12. Mach number averaged over the throat as a function of time for full-order and reduced-order models. Top: control off. Bottom: control on. $T_{0}=1 / f_{0}$

pensive. The reduced-order models were used to generate 100 data points over the frequency range of interest; to generate this amount of data with the original model would be computationally prohibitive.

The data generated by the reduced-order models were used to fit a secondary lower-order model using an optimization technique. The controller transfer function $W_{c}$ was then designed using $H^{\infty}$ optimization: adding a low pass filter to the input path, $\left|\left(G+W_{a} W_{c} W_{s}\right)(i \omega)\right|$ was minimized over the range $\left[0,1.5 f_{0}\right]$. Optimization was made more aggressive over the range $\left[0,1.5 f_{0}\right]$ at the expense of an ill-behaved response beyond $f=1.5 f_{0}$. For more details on the controller design, see Lassaux [14].

The important question is whether the controller designed using only reduced-order dynamics will be effective for the highorder CFD model. The controller was implemented in the fullorder linearized CFD code and comparison was made of the Mach number response to an incoming density disturbance of amplitude $1 \%$ of the nominal value $\rho_{0}$. Figure 12 shows the response $y$ at the throat for both the linearized CFD code and the reduced-order model, when actuation is off and on. The inlet disturbance $d$ has the form described in equation (28), with $\alpha$ set to $0.5 f_{0}^{2}$ and peak time at $5 T_{0}$. The significant frequency content of this disturbance lies in the range $\left[0,0.6 f_{0}\right]$, which fits in the design frequency range of the controller. Figure 13 shows the controller output, that is the fraction of mass flow dumped though the bleed slots, for both the reduced-order and CFD model.

Both the reduced-order and the full-order linearized model yield satisfactory response using the controller design presented previously. The variation in Mach number is considerably reduced by using the controller and the reduced-order model response is virtually indistinguishable from that of the full-order linearized model. This shows that, for this case, the reducedorder models enable control design and accurate simulation of the linearized CFD model response under control. Moreover, 


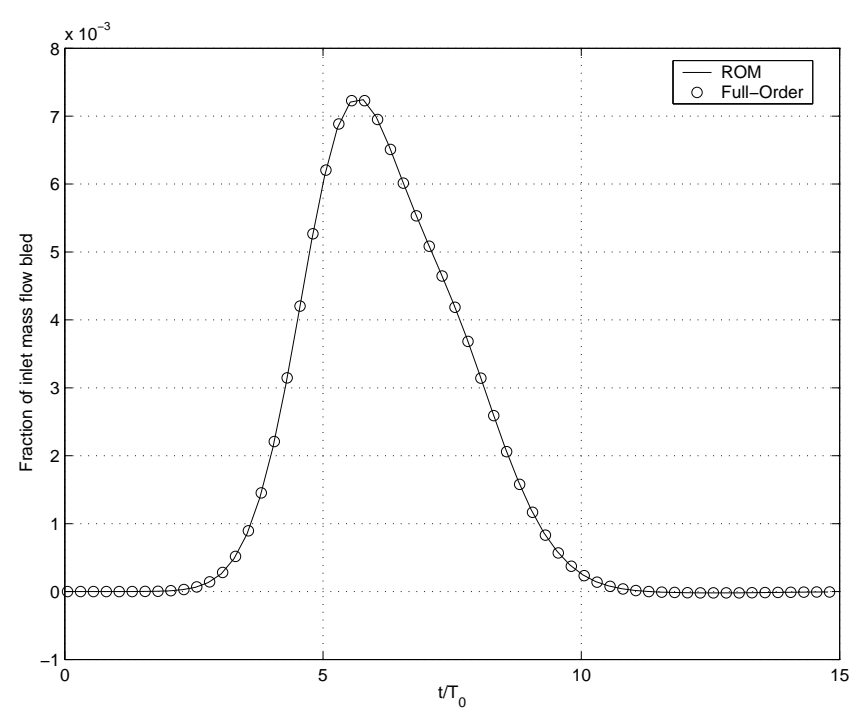

Fig. 13. Fraction of inlet mass flow dumped through upstream slots, for fullorder and reduced-order model.

since the controller is effective at reducing the amplitude of the variation, the linearization assumption of small perturbations should hold for the controlled system.

\section{CONCLUSIONS}

The multiple-point Arnoldi method developed in this paper combines aspects of the traditional Arnoldi approach and the frequency domain POD. The POD-based concept of sampling at multiple frequencies and selecting a basis using SVD leads to efficient reduced-order models, which are accurate over a broad range of frequencies. The Arnoldi-based concept of evaluating multiple vectors at each frequency point reduces the computational cost of the reduction process without a significant compromise in accuracy. By balancing the number of frequency points and Arnoldi vectors per frequency point, the multiplepoint Arnoldi models can achieve roughly the same level of accuracy as POD models with the same number of basis vectors, but with a significant decrease in the cost of obtaining the model.

For the supersonic diffuser considered here, the multiplepoint Arnoldi approach led to reduced-order models on the order of 60 states, which captured the relevant dynamics with a high degree of accuracy. This level of accuracy was quantified by comparing the transfer functions of the reduced and fullorder systems over the range of interest, and by performing a time-domain simulation using both CFD and reduced-order models.

Reduced-order models were developed for all aspects of a supersonic diffuser control problem, which aimed to control the throat Mach number in the presence of an incoming density disturbance using upstream pressure sensing and bleed actuation. These models were use to generate many transfer function data points, which were subsequently processed to obtain a set of very low-order models. These models were then used to design a simple feedforward controller. When implemented on the linearized CFD model, this controller was shown to work effectively. Application of the controller to the nonlinear CFD model is the subject of ongoing research.

\section{ACKNOWLEDGMENTS}

The authors would like to thank Mark Drela, Alex Megretski and Jim Paduano for their very helpful contributions to this research.

\section{REFERENCES}

[1] K.C. Hall, A Linearized Euler Analysis of Unsteady Flows in Turbomachinery, Ph.D. thesis, Dept. of Aeronautics and Astronautics, MIT, May 1987.

[2] J.E. Ffowcs Williams and X. Huang, "Active Stabilization of Compressor Surge," Journal of Fluid Mechanics, vol. 204, pp. 245-262, 1989.

[3] J.D. Paduano, A.H. Epstein, L. Valavani, J.P. Longley, E.M. Greitzer, and G.R. Guenette, "Active Control of Rotating Stall in a Low-Speed Axial Compressor," Journal of Turbomachinery, vol. 115, pp. 48-56, 1993.

[4] E.H. Dowell, K.C. Hall, J.P. Thomas, R. Florea, B.I. Epureanu, and J. Heeg, "Reduced Order Models in Unsteady Aerodynamics," AIAA Paper 99-1261, 1999.

[5] L. Sirovich, "Turbulence and the Dynamics of Coherent Structures. Part 1 : Coherent Structures," Quarterly of Applied Mathematics, vol. 45, no. 3, pp. 561-571, October 1987.

[6] G. Berkooz, P. Holmes, and J.L. Lumley, "The Proper Orthogonal Decomposition in the Analysis of Turbulent Flows," Annual Review of Fluid Mechanics, vol. 25, pp. 539-575, 1993.

[7] W.R. Graham, J. Peraire, and K.Y. Tang, "Optimal control of vortex shedding using low-order models. Part I - Open-loop model development," International Journal for Numerical Methods in Engineering, vol. 44, no. 7, pp. 945-972, 1999.

[8] W.R. Graham, J. Peraire, and K.Y. Tang, "Optimal Control of Vortex Shedding Using Low-Order Models, Part II - Model-Based Control," International Journal for Numerical Methods in Engineering, vol. 44, no. 7, pp. 973-990, 1999.

[9] L.M. Silveira, M. Kamon, I. Elfadel, and J. White, "A CoordinateTransformed Arnoldi Algorithm for Generating Guaranteed Stable Reduced-Order Models of RLC Circuits.," Computer Methods in Applied Mechanics and Engineering, vol. 169, no. 3-4, pp. 377-389, February 1999.

[10] K.E. Willcox, J. Peraire, and J. White, "An Arnoldi approach for generation of reduced-order models for turbomachinery," Computers and Fluids, vol. 31, no. 3, pp. 369-89, 2002.

[11] E. Grimme, Krylov Projection Methods for Model Reduction, Ph.D. thesis, Coordinated-Science Laboratory, University of Illinois at UrbanaChampaign, 1997.

[12] Mark Drela, Two-Dimensional Transonic Aerodynamic Design and Analysis using the Euler Equations, Ph.D. thesis, Massachusetts Institute of Technology, 1987.

[13] Joel R. Phillips, "Automated extraction of nonlinear circuits macromodels," IEEE 2000 Custom Integrated Circuits Conference, 2000.

[14] G. Lassaux, "High-Fidelity Reduced-Order Aerodynamic Models: Application to Active Control of Engine Inlets.," M.S. thesis, Dept. of Aeronautics and Astronautics, MIT, June 2002.

[15] K. C. Hall, J. P. Thomas, and E. H. Dowell, "Reduced-Order Modeling of Unsteady Small-Disturbance Flows Using a Frequency-Domain Proper Orthogonal Decomposition Technique," AIAA Paper 99-0655, 1999.

[16] K.E. Willcox, J.D. Paduano, J. Peraire, and K.C. Hall, "Low Order Aerodynamic Models for Aeroelastic Control of Turbomachines," AIAA Paper 99-1467, 1999.

[17] T. Kim, "Frequency-Domain Karhunen-Loeve Method and Its Application to Linear Dynamic Systems," AIAA Journal, vol. 36, no. 11, pp. 2117-2123, 1998. 